\title{
Glosse Dialettali negli Scholia Omerici
}

\author{
FRANCO MONTANARI
}

Lo studio delle glosse dialettali menzionate negli scholia omerici può essere visto in primo luogo entro un quadro di ricerca ben noto e frequentato, vale a dire quello degli studi degli antichi sulla dialettologia, e messo in connessione con la copiosa produzione lessicografica, nel cui alveo i materiali e le osservazioni di carattere dialettale costituiscono una presenza abbondante: nella lessicografia alessandrina il concetto di dialektos ha un chiaro valore geografico, a indicare le parlate locali di luoghi diversi e specifici, ${ }^{1}$ spesso chiamate in causa nello studio delle opere letterarie, in primis la poesia, come accade negli scholia

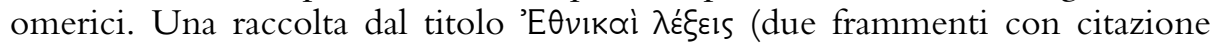
sicura in Galeno, uno possibile in Ateneo) è attribuita a Zenodoto, anche se non sono eliminati i dubbi se si tratti di Zenodoto di Efeso o di un altro Zenodoto e, nel caso di Zenodoto di Efeso, se si tratti di un'opera distinta dalle $\Gamma \lambda \tilde{\omega} \sigma \sigma \alpha$ l oppure no. Scrive K. Nickau: «Gehörte das Werk, wie unsere Vermutung lautet, dem Z(enodotos) von Ephesos und erklärte er sowhol Dialektwörter als auch homerische Glossen, so läßt sich seine Stellung in der Geschichte der Griechischen Lexikographie wie folgt verstehen: Philetas hatte die dunklen Wörter ... gesammelt, vielleicht auch, um den Schmuck des seltenen Ausdrucks (im Sinne von Aristot. poet. 22, 1545 a 9; vgl. Latte, Kl. Schriften 654) den Dichtern verfügbar zu machen, und $Z$ (enodotos) mag ihm darin gefolgt sein. Ebenso wie Philetas erklärte $Z$ (enodotos) Dialektwörter und Homerglossen, doch den Ephesier führten seine Homerstudien whol noch stärker zur Deutung schwer verständlicher Wörter in Ilias und Odyssee». ${ }^{2}$ Accostiamo una efficace sintesi di R. Pfeiffer: "There was hardly any following age in which the Greek mind was not attracted by this problem of explaining $\lambda \dot{\varepsilon} \xi \varepsilon 1 s$. Their origin and their changes, the differentiation of kindred words, the comparison between Greek dialects or between Greek and foreign words were discussed by the Sophists, by Democritus, and by the great Attic philosophers. In the new era the poets revived these studies with fervour, not only Philitas and Simias, but also Callimachus and Apollonius ... Whatever had been undertaken piecemal here and there in the course of time, was all now united into one great enterprise, the $\Lambda \dot{\varepsilon} \xi \varepsilon 15$ of Aristophanes. A collection of $\gamma \lambda \tilde{\omega} \sigma \sigma \propto l$ was

1 Cfr. Cassio 1993a, 1993b, pp. 79 sgg., 1997; Tosi 1994, partic. p. 209; Cassio 2008, pp. 3-8.

2 Nickau 1972, 40-42, cit. da col. 42. 
usually limited to obsolete and obscure terms; but under the neutral title $\Lambda \varepsilon ́ \xi \varepsilon 1 \varsigma$ every word which was peculiar in form or significance and therefore in need of explanation could be listed, whether it was out of date or still in use». ${ }^{3}$ Pfeiffer ricorda poi due punti a suo avviso centrali del lavoro di Aristofane: l'attenzione per lo sviluppo della lingua con la distinzione cronologica fra uso antico e moderno, con l'idea della possibile origine locale di quest'ultimo; un

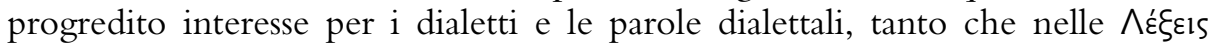

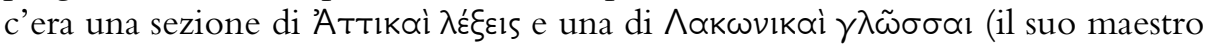

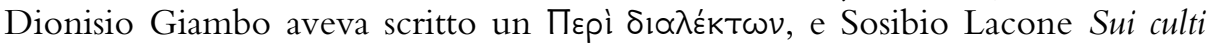
lacedemoni, con raccolta di glosse laconiche). ${ }^{4}$

Nell'ambito della filologia e della grammatica di età ellenistica e imperiale, gli studi dialettologici furono molto praticati e si svilupparono in connessione con problematiche quali la correttezza linguistica e la facies dialettale degli autori dei quali si doveva stabilire e interpretare il testo. Una figura importante in questo quadro è quella del grammatico Demetrio Issione, allievo di Aristarco

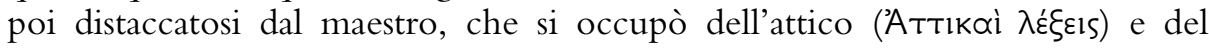

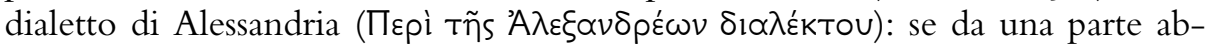
biamo un dialetto con il quale era impossibile non fare i conti, data la produzione letteraria interessata, dall'altra abbiamo con ogni probabilità uno sviluppo dell'interesse per la lingua parlata contemporanea. ${ }^{5}$ Oltre ai vari trattati o raccolte dedicati a singoli dialetti (fra cui un curioso 'I $\tau \propto \lambda_{1 \kappa} \propto \grave{l} \gamma \lambda \hat{\omega} \sigma \sigma \propto l$ di Diodo-

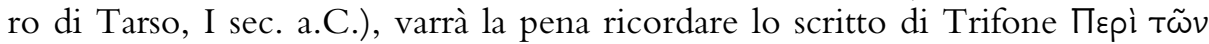

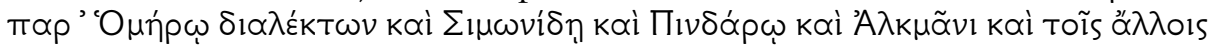
$\lambda$ ирıкоі̃s, dove spicca l'attenzione da una parte per Omero, dall'altra per i poeti lirici, la cui variegatezza e complicazione dialettale poneva problemi talvolta assai ardui. ${ }^{6}$ È una linea alla quale possiamo dedicare solo un breve cenno riassuntivo, sottolineando come essa dirami i suoi rivoli almeno in tre direzioni: la lessicografia, di cui già si è detto; lo studio linguistico-grammaticale, che, dopo le epocali sistemazioni di Apollonio Discolo ed Erodiano, per questo aspetto

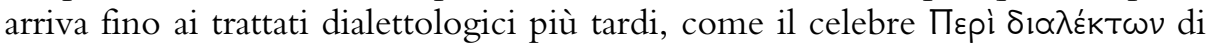
Gregorio di Corinto (X-XI sec.); la filologia e l'interpretazione dei testi, che è quello che ci interessa primariamente in questa sede.

3 Pfeiffer 1968, pp. $197 \mathrm{sg} .=$ pp. $311 \mathrm{sg}$.

4 Pfeiffer 1968, pp. 201 sg. = pp. 316 sg. Pfeiffer vede in Aristofane, oltre a una coscienza dello sviluppo diacronico della lingua, anche un interesse - evidentemente collegato - per la lingua parlata del suo tempo. Il problema nel suo insieme è stato discusso da Callanan 1987, pp. 75-82, da Ax 1990, pp. 13-15: Callanan nega recisamente la presenza in Aristofane di interessi per lo sviluppo diacronico della lingua, ma questa posizione è sembrata eccessiva e infondata ad Ax, anche sulla base di documentazione fornita dagli scholia omerici; si veda da ultimo Pagani 2011, pp. 37 e n. 81, 48 e n. 121.

5 Forse di ascendenza aristofanea, cfr. n. 4; su Demetrio Issione vedi Ascheri 2009 e Ascheri 2010, con utili sintesi e informazioni bibliografiche; sul dialetto di Alessandria vedi Fournet 2009.

6 Cfr. opp. citt. alla n. 1. 
Nella storia dell'interpretazione della poesia nel mondo antico, la Poetica di Aristotele è un testo chiave, nel quale si trova una riflessione su tutto il periodo precedente e si gettano decisive fondamenta per l'attività filologica ed ermeneutica dei secoli successivi. Prendiamo le mosse da ben noti passi della Poetica di Aristotele.

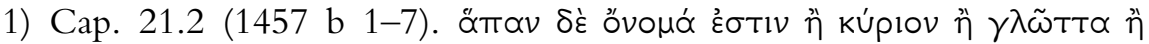

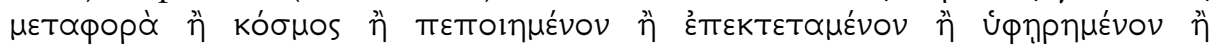

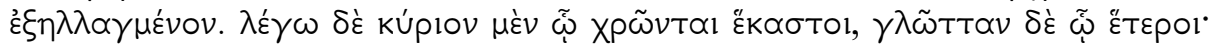

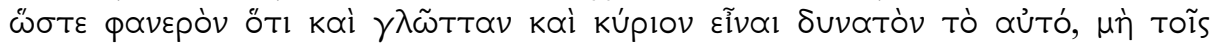

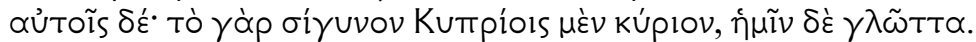

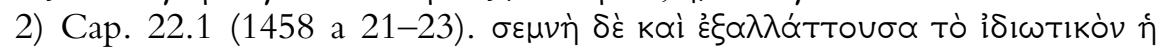

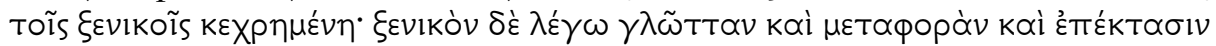

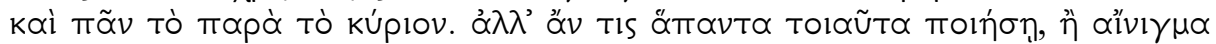

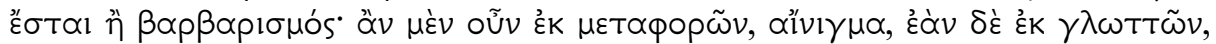

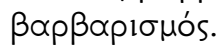

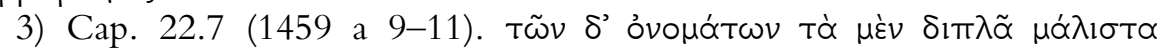

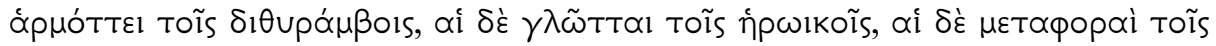
i̊ußzíors.

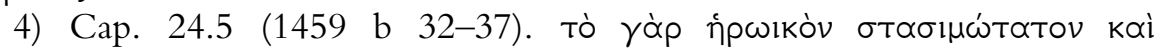

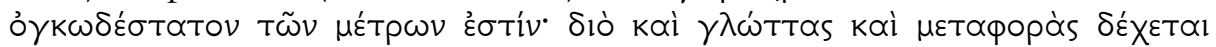

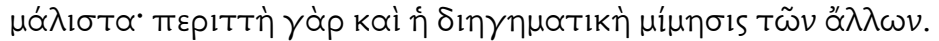

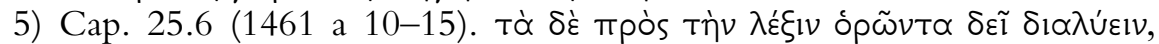

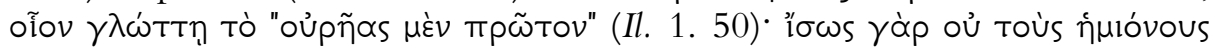

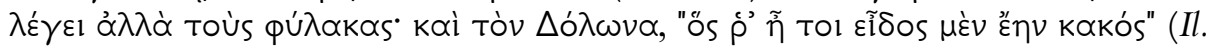

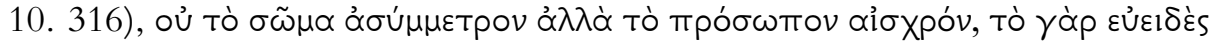

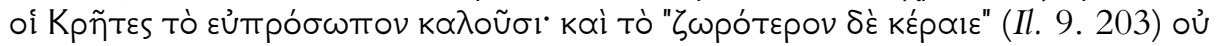

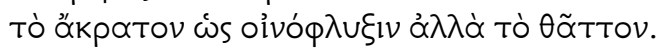

Alla fine del cap. 19 della Poetica, Aristotele introduce la trattazione della lexis (1456 b 9), che si sviluppa poi a partire dal cap. 20 (1456 b 20 sgg.). Nel cap. 21 (passo nr. 1) abbiamo la definizione generale del concetto linguisticolessicale di glossa: il termine designa un onoma usato da "altri" rispetto a quello di uso comune per tutti, cioè differente rispetto a ciò che è dominante. Nel passo nr. 2 la glossa è coerentemente inclusa in un insieme definito §ॄvikóv,

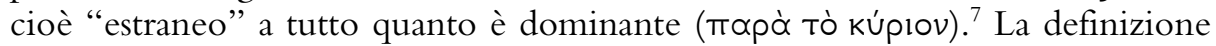
ha una portata del tutto generale e comprende vari tipi di "alterità", precisabili principalmente come di ordine temporale (una parola desueta rispetto al momento in cui viene usata) oppure geografico-dialettale (una parola di area geografica diversa rispetto a quella in cui viene usata): emergerà in seguito (passo nr. 5) che la glossa risulta un elemento oscuro del discorso e quindi ha bisogno di esegesi. Segue l'importante precisazione che lo stesso elemento lessicale può essere sia glossa sia dominante, ma non per le stesse persone, cioè non nello

7 Lucas 1972, p. 208, ad loc; Barabino 2010. 
stesso periodo e nello stesso luogo. L'esempio addotto si basa sull'aspetto geografico-dialettale e non su quello cronologico: la parola oíruvov appartiene al dialetto cipriota (nel senso di "lancia", come già si legge in Erodoto V 9. $3^{8}$ ), per cui a Cipro essa costituisce un elemento linguistico kúprov, mentre è glossa "per noi", scil. gli Attici.

Il cap. 22 è dedicato a definire i pregi del linguaggio poetico, che deve essere elevato e diverso dal linguaggio comune grazie all'uso di elementi estranei a quest'ultimo (cfr. sopra e passo nr. 2). Tutte le espressioni estranee al modo di parlare dominante conferiscono allo stile un carattere non comune e volgare, però non bisogna esagerare perché l'eccesso di glosse, metafore, abbellimenti vari può nuocere alla comprensione (mentre la parola comune è il fondamento della chiarezza: 22.2, 1458 a 34 e 1458 b 2-5), e addirittura condurre al ridicolo $(22.4,1458$ b 13-14): Aristotele invita a considerare l'epica come esempio di uso appropriato di tali pregi del linguaggio (1458 b 15-16). In seguito, nei passi nr. 3 e nr. 4 Aristotele afferma che l'uso di glosse è particolarmente adatto all'epica: nel nr. 3 si parla soltanto di glosse, nel nr. 4 di glosse e anche di metafore, che nel nr. 3 sono riservate alla poesia giambica, ma un collegamento specifico glosse - epica (tò ípwıkóv) è costante e ben sottolineato, anche se ovviamente in nessun modo esclusivo, dato che coinvolge fortemente anche lo stile della tragedia, come risulta tra l'altro dall'esemplificazione di 22.4-5 (1458 b $11-1459$ a 9 ).

Il cap. 25 illustra una serie di criteri esegetici, che possono essere utilizzati per interpretare le difficoltà dell'espressione poetica. All'inizio del cap. Aristotele anticipa che, poiché gli oggetti della mimesis vengono comunicati per mezzo del linguaggio, bisogna prendere in considerazione le glosse, le metafore e i $\pi \circ \lambda \lambda \grave{\alpha} \pi \alpha \dot{\alpha} \theta \eta$ Tñs $\lambda \varepsilon_{\varepsilon}^{\prime} \xi \varepsilon \omega s$. L'argomento è sviluppato poco più avanti (passo nr. 5), adducendo come esempi tre espressioni omeriche nelle quali un problema esegetico può essere risolto, secondo Aristotele, assumendo che una parola difficoltosa sia utilizzata in un senso obsoleto o dialettale: così si eliminano problemi di illogicità, di incongruenza o di sconvenienza. Non ci soffermiamo sulla validità dei problemi esegetici presentati e delle soluzioni avanzate. ${ }^{9}$ Per il nostro discorso serve osservare come la lysis del secondo esempio comporti il

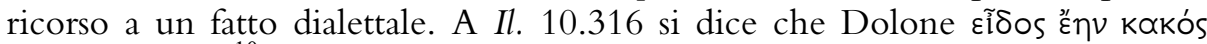

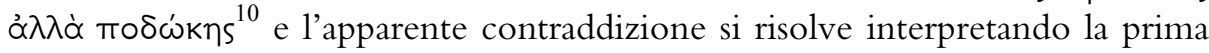
espressione non nel senso che Dolone fosse deforme nel corpo, che sarebbe in contraddizione con il fatto di essere un buon corridore, bensì nel senso che era brutto in viso: si tratta di un uso dialettale di Creta, sostiene Aristotele, dal

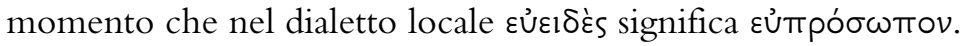

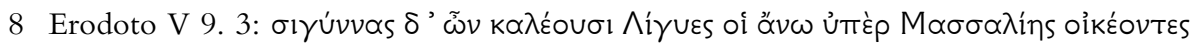

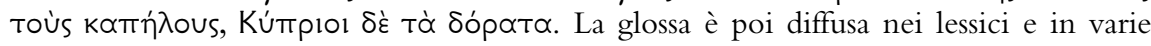
fonti erudite.

9 Vedi i comm. ad loc. di Bywater 1909, Gudeman 1934, Lucas 1972, Gallavotti 1974.

10 La clausola del verso non è riportata nel testo pervenuto della Poetica. 
Nell'ambito della trattazione sulla lexis, Aristotele definisce dunque la dottrina della "glossa", intesa come elemento stilistico proprio del linguaggio poetico, con particolare riferimento all'epica (e alla tragedia: cfr. cap. 25. 4-5, 1458 b 11 - 1459 a 3). ${ }^{11}$ Il tema si ritrova nella parte iniziale del III libro della Retorica (capp. 1-3), con dichiarati rimandi alla Poetica (1404 b 7, 1404 b 28, 1405 a 5). Nell'indicare in parallelo la diversità fra lo stile della prosa e quello della poesia, Aristotele chiarisce che gli ornamenti ottenuti grazie a elementi che si discostano dall'uso comune non sono in genere adatti al linguaggio pro-

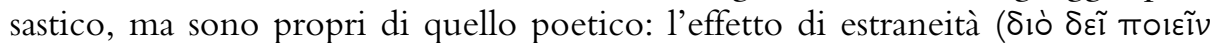

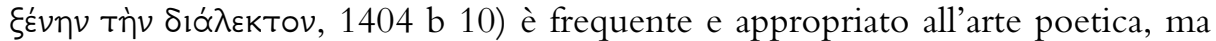
assai meno alla prosa, tanto più che una qualità essenziale dello stile è la chiarezza, che risulta soprattutto da ciò che è kúplov. ${ }^{12}$ Fra gli ornamenti del lin-

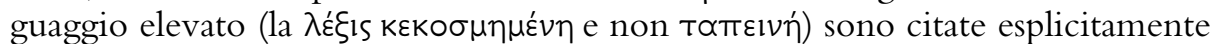
anche le glosse (il termine si trova in 1404 b 28, 1406 a 6, 1406 b 2). Aristotele evidenzia come ci sia stata un'evoluzione nello stile della prosa: «Nacque per primo uno stile poetico, come per esempio quello di Gorgia, e ancora oggi la maggior parte delle persone incolte ritiene che siano gli oratori di questo genere a parlare meglio, mentre non è così, e lo stile della prosa è diverso da quello della poesia»; anche nella tragedia si riscontra una tendenza nella direzione del discorso comune, sia nel passaggio dal tetrametro al giambo (più vicino alla lingua parlata), sia nel fatto che $\mathrm{i}$ tragediografi «hanno anche eliminato le parole

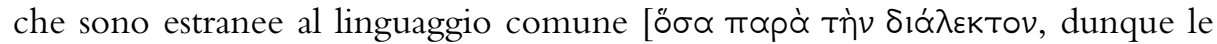
glosse], ${ }^{13}$ grazie alle quali i poeti precedenti abbellivano le loro opere e delle quali tuttora si servono i poeti che compongono esametri» (1404 a 25-35). Assieme all'aspetto di “arcaicità" dell'uso della glossa, ritorna il legame stretto (si direbbe in controtendenza rispetto alla linea prevalente) con la poesia epicoesametrica, legame ribadito a 1406 b 2 con l'affermazione che le glosse sono particolarmente adatte ai poeti epici (oí દ̇тотооюí).

Aristotele non inventa dal nulla il problema della necessità di spiegare le parole difficili nelle opere poetiche e neppure l'uso per esse del termine "glossa". Pfeiffer ha molto ben evidenziato i significativi precedenti della trattazione aristotelica, per cui basta qui un piccolo promemoria. Credo che egli abbia assolutamente ragione quando afferma che già i rapsodi, nel recitare i poemi, dovevano trovarsi nella necessità di offrire embrionali forme di interpretazione, fra cui la spiegazione delle parole difficili di uno stile certamente assai estraneo rispetto al linguaggio comune del loro pubblico, e che di questa più antica

11 Pfeiffer 1968, pp. 12, 75-79 = pp. 55, 142-147.

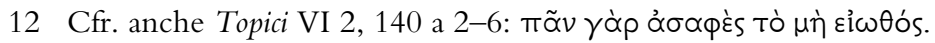

13 Poco più avanti dice che Euripide ha indicato la strada di utilizzare nella composizione poetica le parole della lingua corrente (1404 b 24-25). 
esegesi si trovano tracce nei poeti arcaici e classici. ${ }^{14}$ Pfeiffer mette in evidenza, da questo punto di vista, il significato dell'interesse dei Sofisti per la lingua e degli studi dedicati ai fenomeni linguistici. Fra questi, Prodico si distinse per le sue riflessioni su problemi della lingua e in particolare sui sinonimi e le distinzioni di significato, che - dice Pfeiffer - lo portarono «alla consapevolezza dell'uso diverso nelle diverse parti del paese», tanto che riteneva che Pittaco non sapesse ben distinguere le parole in quanto Lesbio e educato in una lingua straniera (Plat., Prot. $341 \mathrm{c}$ ); inoltre «Platone nel suo Cratilo [401 c] pare abbia

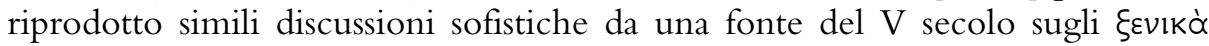
óvómata, quando egli riporta parole eoliche o doriche come 'straniere', cioè diverse dalla famigliare forma attica». ${ }^{15} \mathrm{Nel}$ dossier che stiamo evocando per

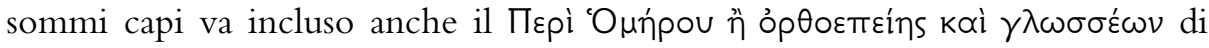
Democrito, opera di cui peraltro può essere dubitata anche la forma del titolo. ${ }^{16}$ Infine, è ben noto il fr. 233 PCG dei $\Delta \alpha \mathrm{l} \tau \alpha \lambda \tilde{n} s$ di Aristofane (commedia rappresentata nel 427 a.C.), ${ }^{17}$ nel quale si trova una delle più antiche testimonianze sull' esegesi omerica e in particolare sulla pratica di spiegare le parole difficili nell'ambito del sistema educativo ateniese del V secolo. Ci sono problemi di ricostruzione puntuale del frammento, ma il senso generale appare acquisito: a un giovane viene chiesto (a quanto pare dal padre) di spiegare e interpretare alcune "glosse" omeriche ( $\left.\lambda \varepsilon \dot{\varepsilon} \xi \circ v^{~ ' O \mu n ́ p o u ~} \gamma \lambda \omega^{\prime} \tau \tau \propto s\right)$; a questo segue la richiesta di spiegare una parola forse di Solone e poi un'altra da un'opera ignota. ${ }^{18}$ Abbiamo dunque due glosse omeriche e due di altra provenienza, per le quali si pone una questione di lessico arcaico e obsoleto.

In sostanza, ci sono tutti gli elementi per essere certi che Aristotele codifica come metodo esegetico e come criterio di analisi stilistica una prassi abbia variatamente diffusa, non solo a livello dell'educazione dei giovani, ma anche in ambiti culturali di diverso tipo, dall'attività dei rapsodi alle riflessioni linguistiche dei filosofi. Ma è importante sottolineare il fatto che lo strumento esegetico si basa, e non potrebbe essere altrimenti, sull'osservazione di una caratteristica essenziale e distintiva del linguaggio dell'epica omerica, e di conseguenza, anche se magari in misura diversa, di quello degli altri generi poetici "alti",

14 Pfeiffer 1968 , pp. $4-5,12,79=$ pp. 45-46, 55, 147; sulle più antiche tracce nei poeti di interpretazione di parole problematiche cfr. anche Montanari 1976, pp. 208-210, con bibliografia.

15 Pfeiffer 1968, pp. 37-43 = pp. 91-99: le citazioni sono a p. $41=96$, dove egli ricorda anche che Erodoto, I 142. 3-4, distingue varianti dello Ionico parlate in diverse città (vedi Asheri 1988, p. 348, ad loc.).

16 Pfeiffer 1968, pp. 42-43 e $79=$ pp. 97-99 e 147.

17 Cfr. Pfeiffer 1968, pp. 14-15 e 79 = pp. 59 e 147; Cassio 1977, pp. 75-77.

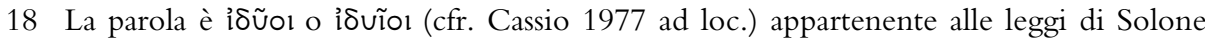
secondo Elio Dionisio 14 Erbse (citato da Eustazio 1158, 20): per la precisione Elio Dionisio la attribuisce a Dracone e a Solone, Fozio, Lex. 136 al solo Solone (fr. 41 b Ruschenbusch); si tratta evidentemente di un termine del linguaggio giuridico. Cfr. Montanari 2003. 
fino naturalmente alla tragedia. Anzi, poiché in ordine logico, e in base a una regola generale, l'osservazione del fenomeno è il fondamento da cui consegue la legittimità di uno strumento esegetico, è consentito chiamare in causa la glossa nell'interpretazione della poesia per il fatto che la glossa stessa è un riconosciuto elemento espressivo dello stile e del linguaggio poetico.

Proviamo ora a seguire specificamente la linea della glossa in quanto elemento estraneo per motivi dialettali, ricordando che Aristotele non ha mancato di precisare che lo stesso elemento lessicale può essere sia glossa sia dominante, ma non per le stesse persone, cioè non nello stesso periodo e nello stesso luogo. Fra gli esempi di glosse addotti da Aristotele nella Poetica, due sono chiaramente indicate come di carattere dialettale: 1) la parola oíruvov appartiene al dialetto cipriota, per cui a Cipro è un elemento linguistico kúpiov, mentre per gli Ateniesi è una glossa; 2) in Il. 10. 316 عĩoos kokós significa che Dolone era brutto in viso e non deforme nel corpo: si tratta di un uso dialettale di Creta,

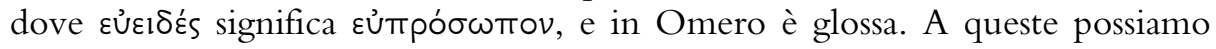
aggiungere la parola costituita da un triplice composto, citata all'inizio del cap. 21 (1457 a 35) come in uso a Marsiglia: le parole composte sono anch'esse un elemento del linguaggio poetico elevato e direi che qui possiamo parlare di una parola composta che è anche una glossa per motivi dialettali.

Se ci volgiamo ora finalmente agli scholia omerici, possiamo come primo passo provare a gettare uno sguardo, anche cursorio, all'Index V: Res potiores, imprimis grammatica et rhetorica dell'edizione degli Scholia in Iliadem di H. Erbse, s.v. Dialecti (vol. VII, pp. 84-88). La quantità di materiali elencata è assai grande e toglie subito l'illusione di analizzarla tutta per un'occasione come questa, specie se all' Iliade si volesse aggiungere anche l'Odissea. Alla ricerca di un punto di partenza nel mare magnum, un'indagine che possiamo fare è verificare se nella scoliografia omerica conservata troviamo la menzione di glosse dialettali attribuite alle due aree geografiche citate nella Poetica, vale a dire Cipro e Creta. L'avvio è un po' occasionale, ma il risultato mi pare di un certo interesse. Cominciamo dal fatto che negli scholia omerici ho trovato 13 glosse attribuite al dialetto cipriota: tutte riguardano l'Iliade.

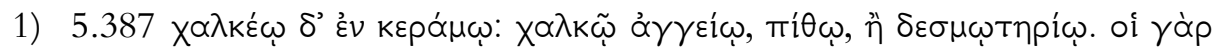

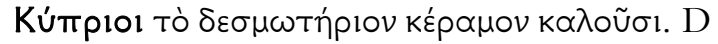

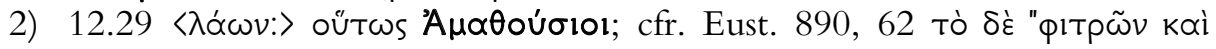

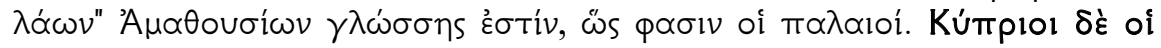

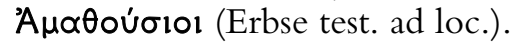

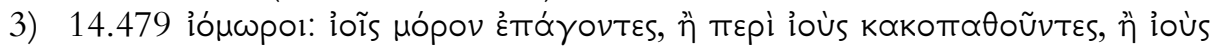

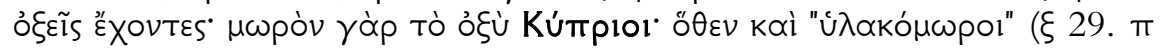
4).

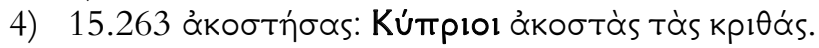

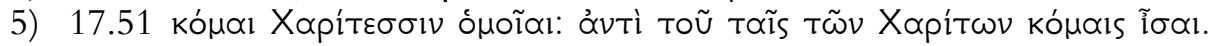

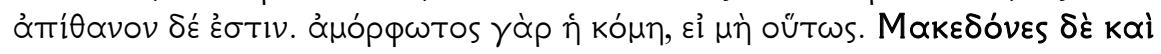

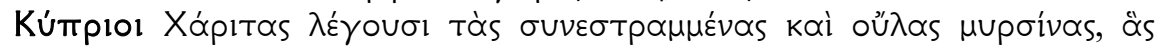


$\phi \propto \mu \dot{\varepsilon} v \sigma т \varepsilon \phi \propto v i ́ t 1 \delta \propto s . \mathrm{D}$

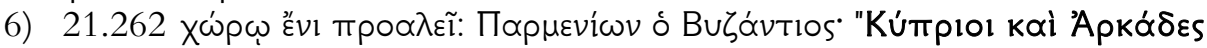

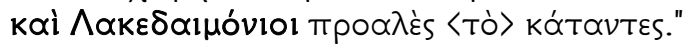

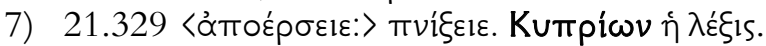

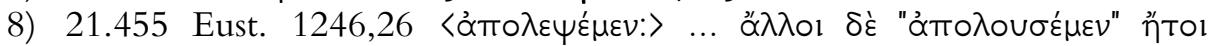

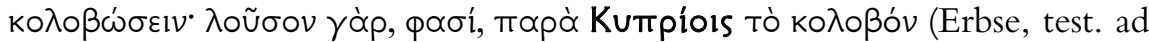
loc.).

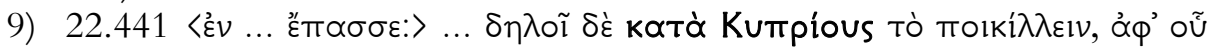

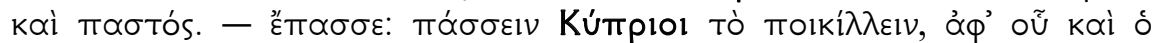
Tळótós. ${ }^{19}$

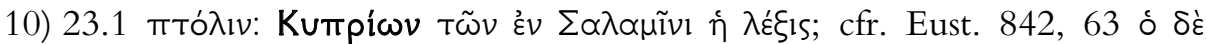

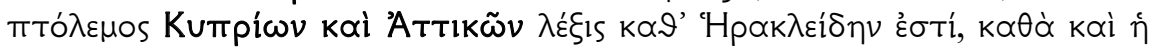
$\pi т \hat{\lambda}_{15}$ (Erbse test. ad loc.).

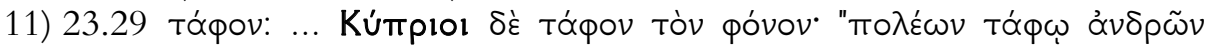

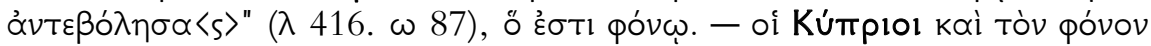

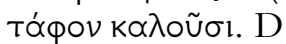

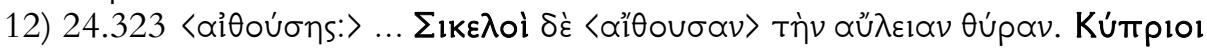

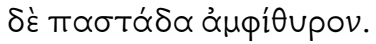

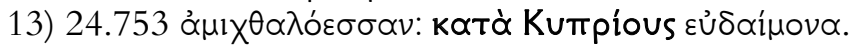

Non possiamo soffermarci su tutti questi casi, facciamo solo qualche osservazione sparsa. Nel nr. 1 la possibile glossa cipriota aggiunge una sfumatura al significato del passo: la straordinarietà dell'imprigionamento di Ares da parte degli Aloadi, Oto ed Efialte, dentro un grande vaso di bronzo ne risulta intensificata se la parola usata dal poeta per indicare il vaso, come glossa cipriota può significare anche $\delta \varepsilon \sigma \mu \omega t \eta \dot{p}$ iov, "prigione".

Nel nr. 2 osserviamo che il riferimento al dialetto cipriota è precisato con il rimando alla parlata specifica della città di Amatunte.

Nel nr. 3 ióuwpos viene comunque ricondotto a lós "dardo", ma per la se-

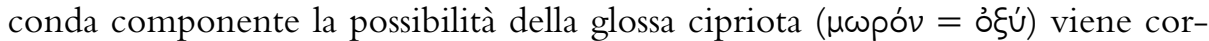
roborata con due rimandi interni all'epica, cioè a un aggettivo usato due volte nell'Odissea.

Nel nr. 4 il valore del verbo ớkoбтóc usato per il cavallo che si nutre alla greppia è interpretato sostenendo che ớkoбtń per "orzo" è parola cipriota. Altrove però la stessa parola è indicata come tessalica: in sch. 6.506-8 leggiamo

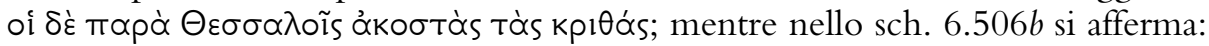

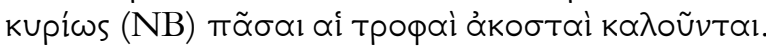

Nel nr. 8 la glossa cipriota è invocata per interpretare la variante

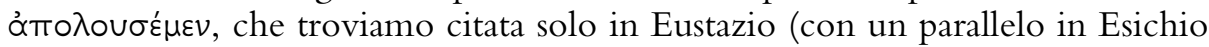
$\propto$ 6465), ma è del tutto assente nella tradizione diretta e anche negli scholia.

19 Nello stesso verso si trova la parola $\theta$ póva, per la quale lo sch. a Teocrito 2, 59-62,

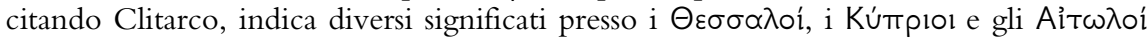
(cfr. Erbse, Sch. Iliad., test. ad loc.). 


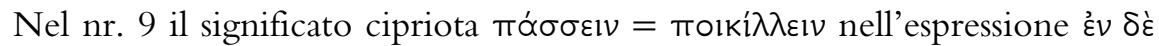

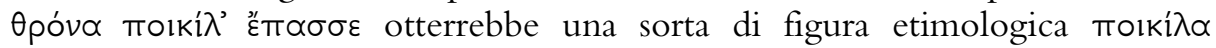

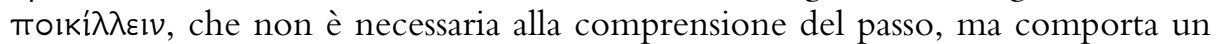
certo impreziosimento stilistico.

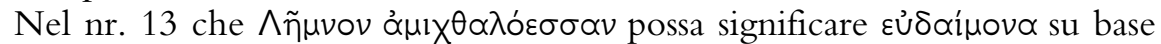
cipriota è una possibilità addotta per spiegare un epiteto di significato incerto (cfr. LfrE s.v.).

In quattro degli esempi citati, assieme al cipriota vengono chiamati in causa altri dialetti: nel nr. 5 il macedone, nel nr. 6 l'arcadico e il lacedemone, nel nr. 10 l'attico, nel nr. 12 il siciliano. Dell'attico avremo occasione di parlare più avanti, ora facciamo qualche rapida considerazione sugli altri dialetti menzionati.

Macedone. La parola ớtta è indicata come macedone nello sch. a Od. 16.31, come tessalica nello sch. D a Il. 9.607 (cfr. LfrE s.v.). Nello sch. Il. $20.404 c$ una parola macedone con vocalismo /a/ viene menzionata accanto a un gruppo di parole che si trovano Tapò $\Delta \omega p \_\varepsilon \tilde{\sigma} \sigma$.

Arcadico. Nello sch. Il. 13.390a per l'aggettivo omerico $\beta \lambda \omega \theta \rho \eta ́$ vengono elencati una serie di significati secondo diversi dialetti (cfr. LfrE s.v.): degli Arcadi, dei Beoti, di Magnesia (in Tessaglia, in Caria, in Lidia?), dei Driopi (popolazione di stirpe ionica: Erodoto I 146.1 ecc.), dei Tirreni, di Caristo (in Eubea). Nello sch. Il. 14.385 il significato della parola ơop presso gli Arcadi e gli Etoli viene distinto da quello omerico.

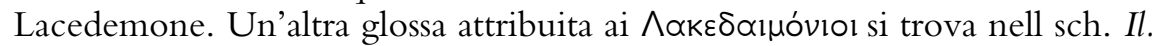

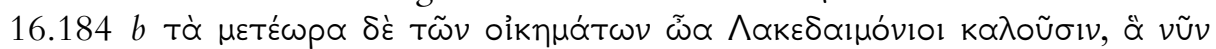

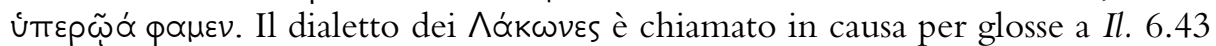

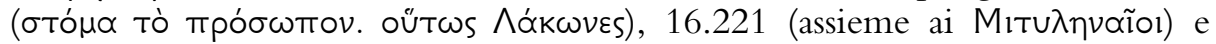
23.83 (^র́kw

Siciliano. Non ho trovato altri riferimenti al dialetto siciliano. Un'affermazione dello sch. Od. 20.383 potrebbe avere anche implicazioni

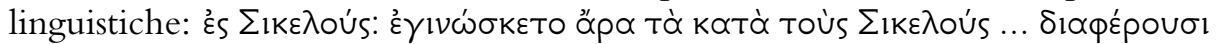

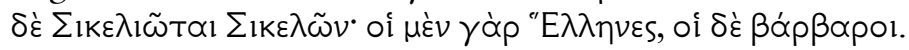

Passiamo ora al dialetto cretese, al quale troviamo riferite 5 glosse, quattro nell'Iliade e una nell'Odissea; non c'è traccia negli scholia della glossa a Il. 10. 316 discussa da Aristotele nella Poetica (cfr. sopra, passo nr. 5, 1461 a 10-15; vd. Erbse ad loc.).

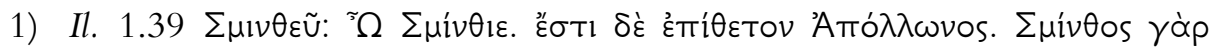

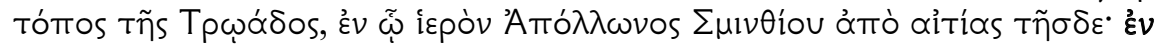

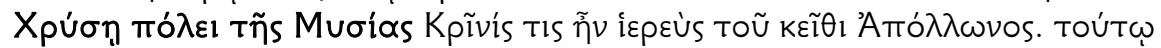

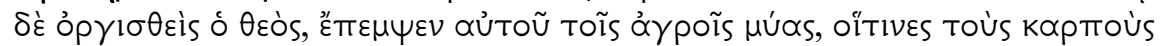

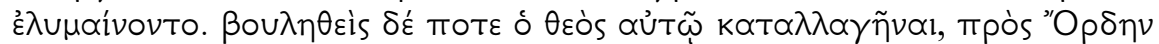

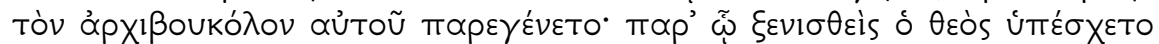

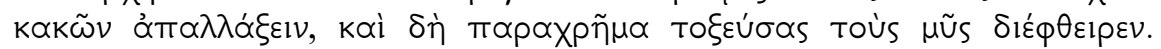

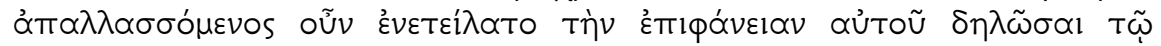




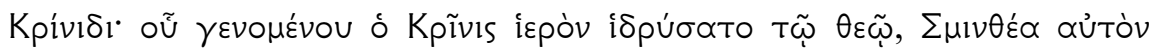

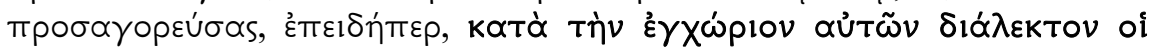

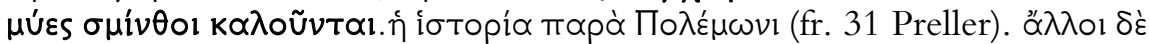
oÚt

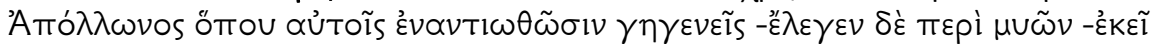

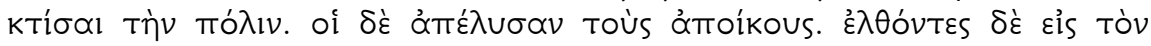

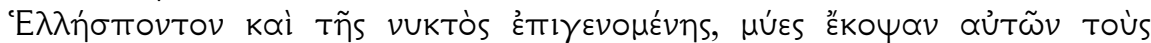

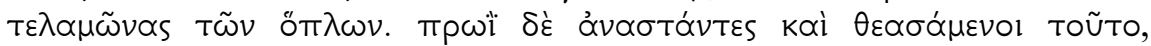

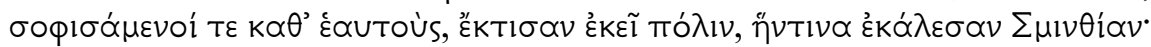

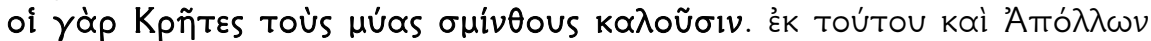

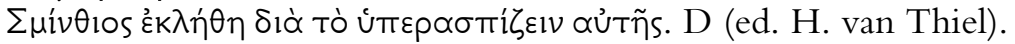

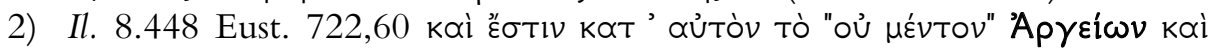

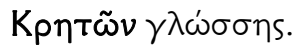

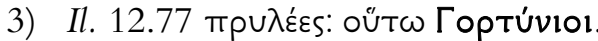

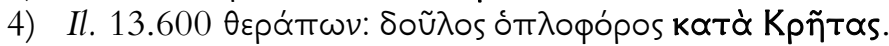

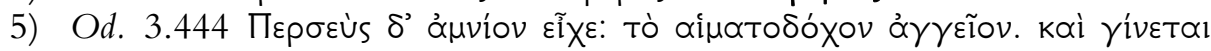

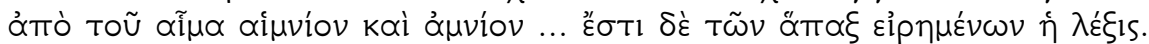

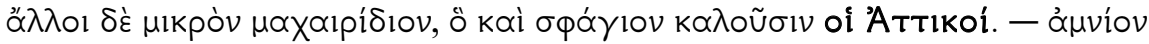

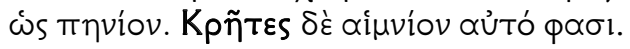

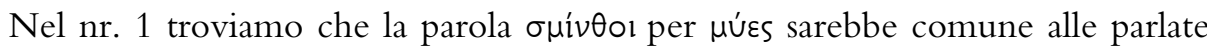
di Creta e della Misia: nel testo omerico essa rappresenta comunque una glossa dialettale, la cui conoscenza è essenziale per capire il significato dell'epiteto con il quale il sacerdote Crise si rivolge ad Apollo. È interessante osservare che, benché per la città della Misia e per Creta siano addotte due storie mitiche differenti, l'epiteto di Apollo viene ricondotto comunque al nome con il quale si indicano $i$ topi nei due dialetti.

Nr. 2: Eustazio (cfr. Erbse, test. ad loc.) è la sola fonte della variante $\mu \varepsilon ́ v$ Tov, risalente a Eraclide di Mileto, per il comunemente tràdito $\mu \varepsilon ́ v \theta \eta \eta$ : si tratterebbe di un'espressione appartenente ai dialetti argivo e cretese.

Nel nr. 3 si identifica una glossa cretese, per la quale si specifica l'appartenza al dialetto di Gortina (cfr. Eust. 893,32; Erbse, test. ad loc.).

Nel nr. 4, per áuvíov il primo significato è "vaso che raccoglie il sangue"

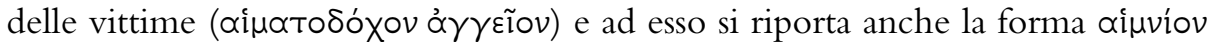

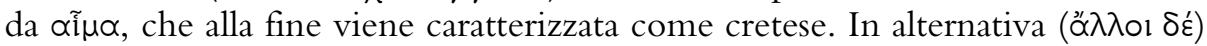
viene menzionato un altro possibile significato, secondo cui åxuviov sarebbe

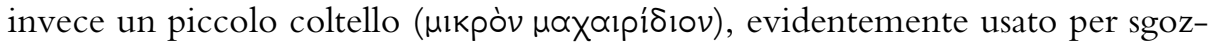
zare la vittima sacrificale; segue un riferimento all'attico, secondo cui questo

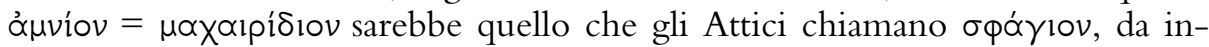

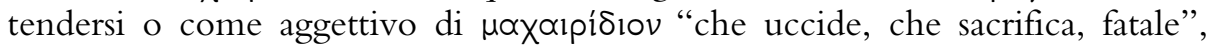
oppure anche come sostantivato "l'uccisore, il sacrificatore". Insomma, le due

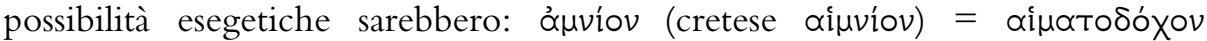

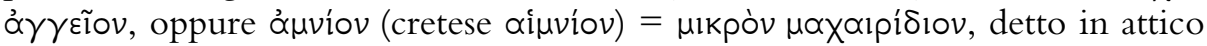
opóy lov. In ogni caso, è certo che questo scolio non parla di una glossa attica 
nella lingua omerica, bensì cita l'equivalente attico di uno dei possibili significati della parola omerica, caso mai molto vicina a un elemento lessicale cretese, utilizzando dunque l'attico come lingua di riferimento.

Questa rassegna è stata già troppo lunga per l'occasione: essa ovviamente non ha altro scopo se non quello di fornire una esemplificazione, che ormai mi pare abbastanza significativa, per quanto limitata. Abbiamo già incontrato per la lingua omerica svariati riferimenti a un notevole numero di "dialetti" appartenenti a diverse aree geografiche, più spesso identificati con il nome del popolo che li parla, più raramente con una indicazione geografica del luogo: cipriota

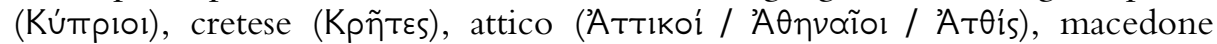

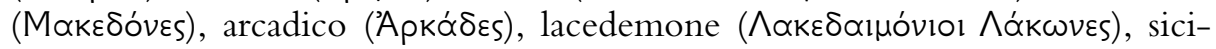

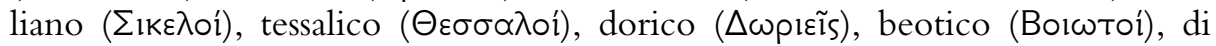
Magnesia (in Tessaglia, in Caria o in Lidia?), dei Driopi (popolazione di stirpe ionica, cfr. sopra), dei Tirreni, di Caristo (in Eubea), di Mitilene, della Misia. Nell'insieme, tenendo presente indici e ricerche operate con il TLG elettronico, vediamo intanto utilizzate denominazioni che vanno da quelle dei grandi

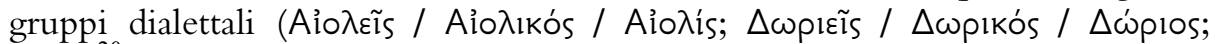

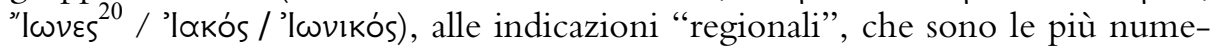
rose (Attica, Tessaglia, Beozia, Cipro, Creta, Macedonia, Etolia ecc.), fino all'individuazione di singole città, come Mitilene, Gortina di Creta o Amatunte di Cipro. Devo confessare che, pur avendo da molto tempo una certa percezione di quanto fosse ricco e variegato il quadro dei riferimenti ai "dialetti" della lingua greca negli scholia omerici e pur sapendo bene che per questo intervento non sarei certo riuscito a esaminare tutto il materiale, quando mi sono messo concretamente al lavoro la realtà si è rivelata ancora più sorprendente delle attese. Oltre a quello che abbiamo sotto gli occhi, dobbiamo considerare due fatti: 1) ciò che rimane nelle raccolte scoliografiche è una piccola parte di quanto fu prodotto dalla filologia antica; 2) la documentazione può essere arricchita da altre cospicue fonti, come il grande e importantissimo mare della lessicografia, Eustazio (che sopra abbiamo citato sporadicamente), altra saggistica omerica di vario genere. Ne risulta senza ombra di dubbio che la quantità e la varietà dei materiali dialettali chiamati in causa dalla critica omerica antica era ampia e abbondante, e porta alla nostra attenzione un fenomeno di straordinario interesse.

Mi limito a menzionare la prospettiva per cui sarebbe certamente interessante seguire e approfondire le possibili implicazioni delle notizie di contenuto dialettale della critica omerica antica a livello di geografia, usi e costumi dei popoli, mitologia. Per indicare un esempio, si può pensare al caso di Il. 1.39, con le due diverse vicende di Apollo collocate una in Misia e l'altra a Creta, e connesse all'epiteto del dio in base al comune nome dialettale dei topi ( $\sigma \mu i ́ v \theta_{0}$ $=\mu u ́ \varepsilon s)$ : Apollonio Sofista 143.9 ci informa che Aristarco collegava l'epiteto

20 lóoves in sch. Il. 13.685. 
con la città di Sminte, nella Troade (dove un culto di Apollo Sminteo esisteva, ma era diffuso anche fuori dalla Troade, peraltro non lontana dalla Misia), e che Apione chiamava in causa una processione Sminteia di Rodi, anch'essa collegata a un intervento di Apollo contro un'invasione di topi.

Lascio da parte la possibilità, o la tentazione, di affrontare questo insieme di dati dal punto di vista della dialettologia greca come è intesa e studiata dalla filologia e linguistica moderna: è un tipo di ricerca che comunque esula ampiamente dalle mie competenze e di cui al momento non mi sento in grado di immaginare le possibilità e i risultati.

Abbiamo evocato all'inizio in estrema sintesi gli elementi del vario e complesso quadro degli antichi studi dialettologici, al quale questi materiali si possono ricollegare. Torniamo ora al punto di vista dello stile e del linguaggio poetico. L'analisi degli antichi delle lingue letterarie dei diversi autori (o anche generi) è ancorata a un principio fondamentale: ogni autore scrive in una lingua essenzialmente e generalmente caratterizzata dall'ambito culturale e geografico principale in cui ha vissuto e operato, nella quale si registra l'innesto e la compresenza più o meno occasionale di elementi presi da dialetti diversi, riutilizzati all'interno di una lingua poetica entro la quale giocano quel ruolo di "glosse" che abbiamo descritto sulle orme di Aristotele: la glossa è un abbellimento dello stile per l'effetto di "estraneità" che comporta; la stessa parola è glossa se inserita in una base di altro dialetto, mentre non è glossa se utilizzata entro una base dello stesso dialetto. ${ }^{21}$

Le testimonianze mostrano che l'individuazione e spiegazione della glossa dialettale può offrire un elemento in più di comprensione del passo, oppure chiarire un aspetto non immediatamente o non del tutto comprensibile: lo sviluppo degli studi dialettologici e l'arricchimento di materiali a disposizione, nei trattati e nelle raccolte, offre al filologo esegeta una strumentazione che diventa sempre più ricca e potente. ${ }^{22}$ Tuttavia, se la base metodologica per l'uso del concetto e per la ricerca della glossa come strumento ermeneutico appare ben chiara, la sua applicazione, almeno in parte, rivela aspetti che lasciano abbastanza perplessi e che, mi pare, invitano a considerare il fenomeno anche da un altro punto di vista.

21 Per questi concetti cfr. da ultimo Cassio 1993a, 1993b, 1997, e le sintesi in Cassio 2008 (soprattutto cap. 3, pp. 70 sgg., e il contributo di C. Vessella, pp. 392-395).

22 Anche per questo aspetto degli sviluppi nell'ambito della filologia alessandrina deve esserci stato un impulso aristotelico-peripatetico. All'osservazione dei dialetti della Grecia e della loro diversificazione orientavano fattori quali: l'interesse per i vari usi e costumi (evidente per esempio nella raccolta delle Politeiai, ricche di dati materiali); lo studio del fatto linguistico in quanto tale e legato alla poesia (Retorica e Poetica); le ricerche, sviluppate dai peripatetici fino dalle prime generazioni, sulle personalità e le opere dei poeti, che portavano in evidenza la diversificazione dialettale. E uno degli aspetti da approfondire a proposito del rapporto tra Aristotele e scuola peripatetica e la filologia ed erudizione di età ellenistica: cfr. Montanari (cur.) 1994, 2000, 2001, 2008; Schironi 2009; Cadoni 2010; sintesi in Montanari, Peripatos, in corso di pubblicazione. 
Non intendo riprendere la discussione dei tre esempi di glosse omeriche addotti da Aristotele nel cap. 25 della Poetica, i cui elementi sono noti. Ricordiamo in breve solo il caso in cui viene menzionato un elemento dialettale,

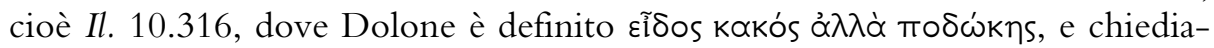

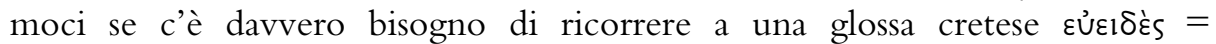
$\varepsilon \cup ̉ \pi p o ́ \sigma \omega \pi$ ov per spiegare come l'espressione non contenga una contraddizio-

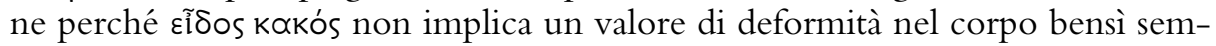
plicemente di bruttezza, che non contrasta per nulla con il fatto di essere un buon corridore.

Di quelli discussi sopra, riprendiamo per esempio il caso di Il. 17.51, dove

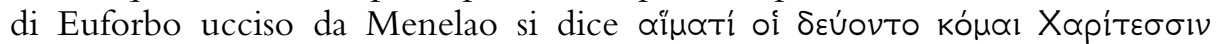

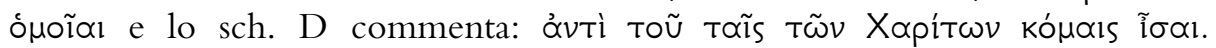

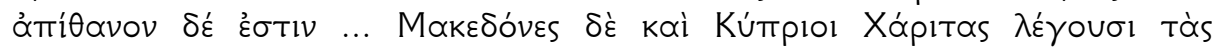

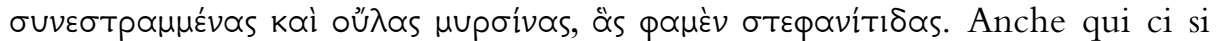
chiede quanto sia "inventato" il problema e il conseguente ricorso a una glossa

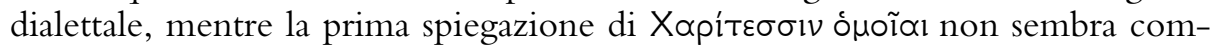
portare alcuna vera difficoltà.

Se l'interpretazione dell'epiteto di Apollo Smintio in Il. 1.39 si giova effettivamente degli elementi dialettali addotti, insieme al racconto delle vicende mitiche che vedono il dio protagonista, bisogna dire che in altri casi l'elemento dialettale invocato per fornire un'interpretazione non appare affatto strettamente o fondamentalmente necessario per capire il passo. Per essere forse un po' brutali, talvolta sembra che ci sia un interesse primario a "inventare" il problema esegetico, a "scoprire" per forza una glossa nel dettato poetico, allo scopo di utilizzare l'elemento dialettale essenzialmente per avere l'occassione di spiegarlo o almeno di arricchire le possibilità esegetiche (si pensi ai casi in cui viene elencata una serie di significati possibili a seconda del dialetto, come per esempio nello sch. Il. 13.390a, citato sopra per l'arcadico). Se talvolta l'elemento dialettale appare effettivamente utile per capire un passo oscuro caratterizzato da una glossa, altre volte esso sembra servire piuttosto per aggiungere al passo una sfumatura che in qualche modo conferisce maggior valore al significato del testo, oppure per offrire un'informazione che riveste essenzialmente un interesse erudito. Allo scopo di perseguire un elemento addizionale che risulta interessante essenzialmente dal punto di vista dell'interprete, sembra profilarsi una sorta di vera e propria "caccia alla glossa" per avere l'occasione di spiegarla, adducendo un dato dialettale con un suo portato di significati aggiuntivi e/o di particolari eruditi. Si direbbe insomma che si vede all'opera una precisa volontà di far emergere ed evidenziare un ricco e inusitato pluridialettalismo omerico, utile anche per quello che porta in più di contorno al testo. A questo fine, il metodo aristotelico della scoperta e analisi della glossa a scopi ermeneutici, lo strumento esegetico messo nelle mani di critici e filologi in base a una precisa analisi del linguaggio e dello stile, subisce un'applicazione indebitamente allargata e estensiva, con il risultato di arricchire il bottino dialettale di un considerevole numero di "scoperte" piuttosto discutibili per quanto riguarda la lingua 
omerica, il che non vuol dire - e questo è un altro aspetto del problema - che siano false o sbagliate sul piano puramente dialettologico in quanto tale. In fin dei conti, quell'idea di mescolanza dialettale nella lingua poetica di ogni autore, fatta di sporadici innesti su una base ben caratterizzata, per il caso particolarissimo di Omero sembra equivalere a una compresenza di tutti i dialetti conosciuti, che si risolve nel pervicace tentativo di ritrovarne ad ogni costo la testimonianza nei poemi. Un'idea che trova espressione in un passo dello ps.

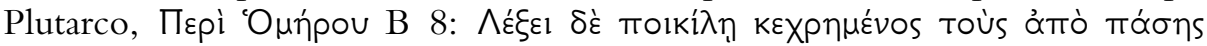

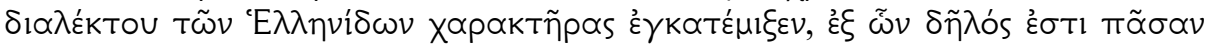

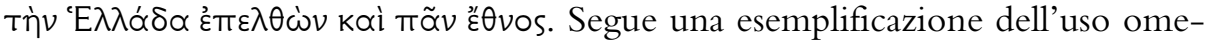
rico di dorico, eolico, ionico, per arrivare all'affermazione (cap. 12) $\mu \alpha^{\prime} \lambda_{l} \sigma \tau \alpha \delta \dot{\varepsilon}$

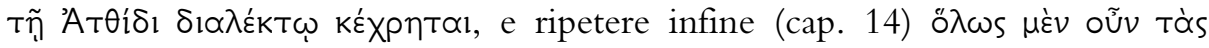

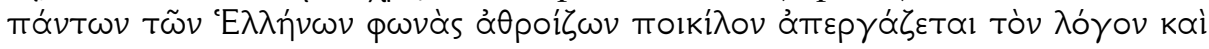

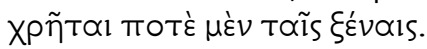

Era Aristarco il sostenitore dell'idea che Omero fosse di origine ateniese e che la lingua di Omero fosse una forma arcaica di Ionico, parlata anticamente in Attica, prima che si diffondesse nelle aree ioniche: nell'uso omerico essa poteva essersi arricchita di tratti dialettali appartenenti ad altre aree della Grecia, che il poeta avrebbe conosciuto nelle sue peregrinazioni. Con questa impostazione, il caso di Omero viene di fatto in qualche modo ricondotto al modello generale: una lingua base caratterizzata dall'ambito culturale e geografico principale in cui il poeta ha vissuto, nella quale si innestano elementi di altri dialetti che funzionano come "glosse". Si tratta peraltro, per il problema della lingua, di una visione coerente con l'impostazione storico-filologica aristarchea per gli altri aspetti e settori dell'omeristica: non si possono attribuire a Omero, per mezzo dell'allegoresi, visioni del mondo e ideologie a lui estranee o posteriori, né gli si possono attribuire conoscenze di qualunque tipo, che si sono manifestate o sono state scoperte in tempi posteriori. Un'analisi storico-filologica altrettanto rigorosa impedisce di attribuire alla lingua di Omero la commistione e la compresenza di tutti i dialetti del mondo greco, vale a dire l'utilizzazione di un impasto linguistico storicamente inspiegabile e inaccettabile. Al rifiuto alessandrino-aristarcheo dell'anacronismo e dell'incoerenza cronologica e storico-culturale si contrapponevano, come è ben noto, tendenze critiche ed ideologiche di segno opposto, capaci di attribuire ad Omero ogni filosofia e religione, e di farne l'origine e il serbatoio di tutte le conoscenze. Quest'ultima idea, nell'ambito della scoliografia, è ben rappresentata nel corpus dei cosiddetti scholia exegetica, mentre è assente negli scholia provenienti dalla linea genuinamente aristarchea del cosiddetto VMK (Viermännerkommentar: Didimo, Aristonico, Nicanore, Erodiano). Gli scholia con riferimenti a glosse dialettali che ho esaminato finora sono tutti scholia exegetica oppure scholia D o passi di Eustazio probabilmente riconducibili a scholia exegetica perduti; ${ }^{23}$ nessuno proviene da VMK.

23 Fenoglio 2009. 
Una diversa testimonianza, tuttavia, si aggiunge al quadro che abbiamo avuto davanti fino a questo punto. Nel suo intervento (pubblicato in questo volume) René Nünlist ha menzionato due scholia di Aristonico che offrono un'informazione dialettale interessante e che vale la pena esaminare.

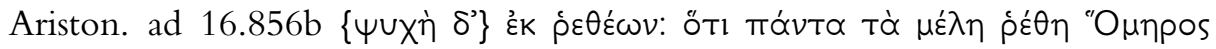

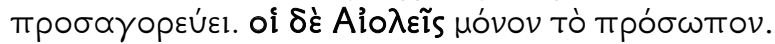

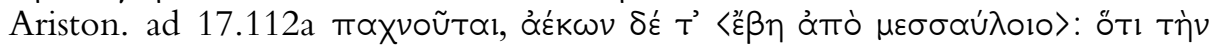

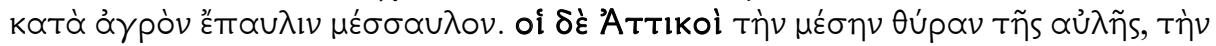

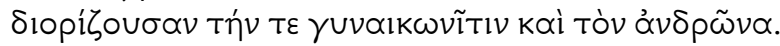

A proposito di 16.856 , si dice che $\rho \dot{\varepsilon} \theta 0$ in Omero indica tutti i $\mu \dot{\varepsilon} \lambda \eta$ del cor-

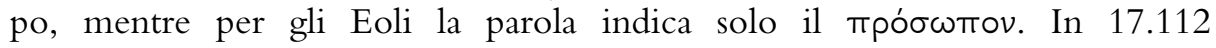
l'osservazione riguarda la diffenza di significato della parola $\mu \varepsilon ́ \sigma \propto v \lambda{ }^{\circ} v$ in Omero e nell'uso attico (scil. posteriore). Si tratta dunque chiaramente di due casi nei quali il dato dialettale non viene addotto per interpretare un qualsiasi aspetto del testo omerico, bensì come una considerazione aggiuntiva di carattere linguistico-lessicale, un'osservazione erudita che non risulta per nulla funzionale all'esegesi. Simili osservazioni possono rientrare nella prospettiva aristarchea, già ricordata sopra, cioè in sostanza risalire allo scopo di evidenziare che la lingua di Omero era una forma arcaica di ionico, parlata anticamente in Attica, diversa dunque per esempio dall'eolico (primo caso) e anche dall'attico (secondo caso). Il fatto che le si trovi in scholia di Aristonico, normalmente improntati a dottrina aristarchea, va in questa direzione, ed è importante proseguire e ampliare la ricerca per vedere se altre testimonianze dello stesso genere possono confortare questa idea.

Per tirare le somme, diremo che l'inusitata e particolare ricchezza di materiali dialettali nei resti della critica omerica antica (di cui quanto conservato non può che essere solo una parte, per di più assai parzialmente esaminata qui) costituisce un fenomeno di fronte al quale si delineano a mio avviso due possibilità interpretative, almeno per il momento.

1) Esso può rappresentare un altro settore che si aggiunge all'idea generale non aristarchea di Omero origine e serbatoio di tutte le conoscenze: anche per la lingua e per i dialetti in Omero si può trovare tutto, ogni cosa di ogni luogo e di ogni tempo, il che serve molto per chi lo interpreta, come in molte altre sfere del sapere.

2) Può trattarsi di tracce risalenti alla visione aristarchea: individuazione (anche grazie all'evidenziazione di differenze specifiche) della base della lingua omerica costituita da una forma arcaica di ionico dell'Attica, arricchita occasionalmente di elementi dialettali variegati e riconosciuti come glosse.

Se dovessi esprimere un'opinione a questo stadio della ricerca, direi che mi sembra più probabile la prima ipotesi per tutte le testimonianze la cui fonte scoliografica è costituita dagli scholia exegetica, mentre è verisimile che la seconda valga piuttosto per i materiali offerti da una fonte di derivazione aristarcheoalessandrina (scholia di VMK, come i due citati di Aristonico). Non mi sento 
affatto di escludere tuttavia che entrambi i fattori abbiano giocato un ruolo in momenti e stratificazioni diversi dell'erudizione omerica e che i loro effetti si siano mischiati nella composita tradizione di un materiale molto miscellaneo, nel quale le osmosi di contenuti non sono certo inusitate. Ciò di cui sono certo è che la ricerca è ben lungi dall'essere terminata: mi accontento di averne impostato $\mathrm{i}$ termini e individuato $\mathrm{i}$ binari sui quali camminare.

\section{Bibliografia}

Ascheri P., Demetrius [14] Ixion, 2009, in LGGA.

Ascheri P., Demetrio Issione "dialettologo": l'attico e il dialetto degli Alessandrini, in: Aner Polytropos. Ricerche di filologia greca antica dedicate dagli allievi a Franco Montanari, a cura di Fausto Montana, Roma 2010, pp. 125-152.

Asheri (cur.): Erodoto. Le storie. Libro I. La Lidia e la Persia, a cura di David Asheri, Milano 1988.

Ax W., Aristophanes von Byzanz als Analogist: zu Fragment 374 Slater (= Varro, de Lingua latina 9, 12), Glotta 68, 1990, pp. 4-18 (rist. in Lexis und Logos. Studien zur antiken Grammatik und Rhetorik, hrsg. von Farouk Grewing, Stuttgart 2000, pp. 116-127).

Barabino A., Una nota sul concetto aristotelico di $\gamma \lambda \tilde{\omega} \sigma \sigma \alpha$, in: Aner Polytropos. Ricerche di filologia greca antica dedicate dagli allievi a Franco Montanari, a cura di Fausto Montana, Roma 2010, pp. 163-170.

Bywater (cur.): Aristotle. On the Art of Poetry, rev. Text with Intr., Transl. Comm. by I. Bywater, Oxford 1909.

Cadoni, N., Aristotele e l'omeristica antica: sondaggi su j̃̈os, in: Aner Polytropos. Ricerche di filologia greca antica dedicate dagli allievi a Franco Montanari, a cura di Fausto Montana, Roma 2010, pp. 3-39.

Callanan C. K., Die Sprachbeschreibung bei Aristophanes von Byzanz, Hypomnemata 88, Göttingen 1987.

Cassio (cur.): Aristofane, Banchettanti, a cura di Albio Cesare Cassio, Pisa 1977.

Cassio (cur.): Storia delle lingue letterarie greche, a cura di Albio Cesare Cassio, Firenze 2008.

Cassio A. C., Alcmane, il dialetto di Cirene e la filologia alessandria, RFIC 121, 1993, pp. 24-36.

Cassio A. C., Parlate locali, dialetti delle stirpi e fonti letterarie nei grammatici greci, in: Emilio Crespo - José Luis García Ramón - Araceli Striano, Dialectologica Graeca. Actas del II coloquio internacional de dialectologia griega, Madrid 1993, pp. 73-90.

Cassio A. C., Futuri dorici, dialetto di Siracusa e testo antico dei lirici greci, AION(filol) 19, 1997, pp. 187-214.

Erbse (cur.): Scholia Graeca in Homeri Iliadem (Scholia Vetera), rec. H. Erbse, voll. I - VII, Berlin-New York, 1969-1988.

Fenoglio S., La riflessione sui dialetti nei Commentarii all'Odissea di Eustazio di Tessalonica, Quaderni del Dipartimento di Filologia, Linguistica e Tradizione Classica “A. Rostagni", n.s. 8, 2009, pp. 239-254. 
Fortenbaugh-Schütrumpf (cur.): Demetrius of Phalerum. Text, Translation and Discussion, Edited by William W. Fortenbaugh - Eckart Schütrumpf, Rutgers Univ. St. in Class. Humanit. (RUSCH), vol. IX, New Brunswich and London 2000.

Fournet J. L., Alexandrie: une communauté linguistique? ou la question du grec alexandrin, IFAO, Le Caire, 2009.

Gallavotti (cur.): Aristotele. Dell'arte poetica, a cura di Carlo Gallavotti, Milano 1974.

Gudeman (cur.): Aristoteles. Пврі поıптıкñs, hrsg. von Alfred Gudeman, Berlin 1934.

LGGA: Lessico dei Grammatici Greci Antichi, diretto da Franco Montanari, Walter Lapini, Fausto Montana, Lara Pagani: http://www.aristarchus.unige.it/lgga/index.php.

Lucas (cur.): Aristotle. Poetics, Introduction, Commentary and Appendixes by D. W. Lucas, Oxford 1968, 1972².

Montanari (cur.): La philologie grecque à l'époque hellénistique et romaine, Entretiens preparés et présidés par Franco Montanari, Entretiens sur l'antiquité classique, Tome XL, Fondation Hardt, Vandoeuvres-Genève, 1994.

Montanari F., Una glossa omerica pre-alessandrina? (Sch. Il. XVI 235 - Eubulo fr. 139 K.), "Rendiconti Istituto Lombardo - Classe di Lettere", 110 (1976), pp. 202-211.

Montanari F., Demetrius of Phalerum on Literature, in: Fortenbaugh-Schütrumpf 2000, pp. 391-411.

Montanari F., Gli studi omerici di Demetrio Falereo, SemRom 4, 2001, pp. 143-157.

Montanari F., I percorsi della glossa. Traduzioni e tradizioni omeriche dall'antichità alla cultura bizantina, in: Erudizione scolastico-grammaticale a Bisanzio, a cura di Paola Volpe Cacciatore, Napoli 2003, pp. 81-88.

Montanari F., Aristotele, Zenodoto, Aristarco e il serpente pietrificato di Iliade II 319, in: Studi offerti ad Alessandro Perutelli, Roma 2008, vol. II, pp. 237-244.

Montanari F., The Peripatos on Literature: Interpretation, Use and Abuse, Prolusione al Convegno "Filosofi della scuola di Aristotele - Cameleonte e Prassifane", Roma, Istituto Svizzero, 5-7 settembre 2007, in corso di pubblicazione nei Proceedings = RUSCH vol. XVIII, forthcoming.

Nickau K., Zenodotos, RE X A, 1972, 19-49.

Pagani L., Pioneers of Grammar. Hellenistic Scholarship and the Study of Language, in: From Scholars to Scholia. Chapters in the History of Ancient Greek Scholarship, Edited by Franco Montanari - Lara Pagani, Trends in Classics - Supplementary Volumes 9, Berlin - New York 2011, pp. 17-64.

Pfeiffer R., History of Classical Scholarship from the Beginnings to the End of the Hellenistic Age, Oxford 1968 = Storia della filologia classica. Dalle origini alla fine dell'età ellenistica, trad. it. Napoli 1973.

Schironi F., Theory into Practice: Aristotelian Principles in Aristarchean Philology, CPh 104, 2009, pp. 279-316.

Tosi R., La lessicografia e la paremiografia in età alessandrina e il loro sviluppo successivo, in Montanari (cur.) 1994, pp. 143-197 + 198-209. 
Bereitgestellt von | SUB Göttingen Angemeldet

Heruntergeladen am | 27.10.14 12:34 